\title{
UPPER AND LOWER BOUNISS FOR EIGENVALUES OF THE LAPLACIAN ON A SPHERICAL CAP
}

\author{
BY \\ FRANK E. BAGINSKI \\ The George Washington University, Washington, D.C.
}

\begin{abstract}
In the following, we derive upper and lower bounds for eigenvalues of the Laplacian on a domain that is a spherical cap whose angular width $2 \vartheta_{0}$ is less than $\pi$. While previous work of this nature seems to focus on the principle eigenvalue, our results apply to any eigenvalue when $0<\vartheta_{0}<\pi / 2$. In addition, some of our results also apply to spherical caps for which $0<\vartheta_{0}<\pi$. When our estimates for the principle eigenvalue are compared to the results of $[4,8]$, we find that our upper and lower bounds are sharper.
\end{abstract}

1. Introduction. The problem of determining the eigenvalues of the Laplacian on a spherical cap arises naturally in a variety of applications. Some of these include the vibrations of spherical shells [7, Ch. 13], diffusion processes on cellular membranes [4], and the buckling of spherical shells [3]. Since the spectrum of the Laplacian varies as a function of the angular width of the cap, it is often necessary to obtain upper and lower bounds for eigenvalues in terms of this parameter. In many cases, the principle eigenvalue $\mu_{1}$ is of most interest and consequently, much work seems to center on determining upper and lower bounds for $\mu_{1}$. For example, when studying boundary value problems, it is well known that the optimal constant in Poincare's inequality is $\mu_{1}$ (i.e., $\|\nabla w\|^{2} \geq \mu_{1}\|w\|^{2}$ where $\|\cdot\|$ is the $L_{2}$ norm (see $[9, \mathrm{p}$. 112])). In [4], the authors were interested in determining the asymptotic behavior of a certain diffusion process which led to the problem of estimating $\mu_{1}$.

There are situations where the eigenvalue of most interest is not the smallest one and estimates are needed for these eigenvalues as well. For example, when studying the buckling of elastic spherical shells, one is interested in the critical buckling load $\lambda_{c}$, the smallest value of the applied force (load) which corresponds to a bifurcation point of the model equations. Although $\lambda_{c}$ is related to the spectrum of the Laplacian, it is not necessarily the principle eigenvalue that plays the key role (see [3]). Problems that relate to ordering the eigenvalues of the Laplacian have been considered in $[1,2]$.

In the work that is presented here, we will focus on deriving upper and lower bounds for any eigenvalue of the Laplacian on a spherical cap for which the angular

Received October 20, 1989.

This work was supported in part by a grant from The George Washington University.

(C)1990 Brown University 
width $2 \vartheta_{0}$ is less than $\pi$. The upper and lower bounds for $\mu_{1}$ that we derive here are sharper than the previous estimates derived in $[4,8]$ for $0<\theta_{0}<\pi / 2$. Although the bounds in $[4,8]$ are valid for $0<\vartheta_{0}<\pi$, they apply only to the principle eigenvalue. Some of our estimates also apply to caps for which $0<\vartheta_{0}<\pi$ (see Sec. $3)$.

In the following, we will let $S_{\vartheta_{0}}$ denote a spherical cap that is parametrized by the usual spherical coordinates $(\vartheta, \varphi)$. In particular, for $(x, y, z) \in S_{\vartheta_{0}} \subset \mathfrak{R}^{3}$, we have

$$
(x, y, z)=(\cos \varphi \sin \vartheta, \sin \varphi \sin \vartheta, \cos \vartheta), \quad 0 \leq \vartheta \leq \vartheta_{0}<\pi, \quad 0 \leq \varphi \leq 2 \pi .
$$

We will consider the following eigenvalue problem on $S_{\vartheta_{0}}$ : Find a real number $\mu$ and a nonzero smooth function $w: S_{\vartheta_{0}} \rightarrow \mathfrak{R}^{1}$ which satisfy

$$
\begin{gathered}
\Delta w+\mu w=0, \quad \text { on } S_{\vartheta_{0}}, \\
w=0, \quad \text { on } \partial S_{\vartheta_{0}},
\end{gathered}
$$

where $\partial S_{\vartheta_{0}}$ denotes the boundary of $S_{\vartheta_{0}}$. It is well known that $\mu$ is an eigenvalue of $(*)$ if and only if $\mu=\nu(\nu+1)$ where $\nu>0$ is a solution of

$$
P_{\nu}^{m}\left(\cos \vartheta_{0}\right)=0 \text {, }
$$

for some $m=0,1, \ldots$, and $P_{\nu}^{m}$ is a Legendre function of the first kind of degree $\nu$ and order $m$ (see [12]). For a general discussion on the Legendre functions and their properties the reader is referred to [5]. For fixed $m$, we will let $\nu=\nu_{j}^{m}$, $j=1,2, \ldots$, denote the positive $\nu$-zeros of (1.1). In the following, $\nu$ will be a positive real, $m, n$ will always denote nonnegative integers and $j, k$ will denote positive integers. The $\nu_{j}^{m}$ 's depend on $\vartheta_{0}$ and in [1] it is shown that $\nu_{j}^{m}=\nu_{j}^{m}\left(\vartheta_{0}\right)$ is analytic in $\vartheta_{0}$.

Corresponding to each $\nu_{j}^{m}$, there is an eigenvalue of $(*)$ which we will denote by $\mu_{j}^{m}=\nu_{j}^{m}\left(\nu_{j}^{m}+1\right)$ with eigenfunction(s):

$$
\begin{array}{ll}
u_{j}^{m}(\vartheta, \varphi)=P_{\nu_{\prime}^{\prime \prime}}^{m}(\cos \vartheta) \cos m \varphi, & m=0,1, \ldots, j=1,2, \ldots, \\
v_{j}^{m}(\vartheta, \varphi)=P_{\nu_{j}^{\prime \prime}}^{m}(\cos \vartheta) \sin m \varphi, & m=1,2, \ldots, j=1,2, \ldots .
\end{array}
$$

From (1.2), we see that the multiplicity of $\mu_{j}^{m}$ is at least 1 when $m=0$ and at least 2 when $m \neq 0$. Properties of the $\nu_{j}^{m}$ 's and $\mu_{j}^{m}$ 's, including examples where an eigenvalue has multiplicity greater than two, are discussed in [1,2].

The major result of this work is Theorem 1 where we establish upper and lower bounds for the eigenvalues of $(*)$ for $0<\vartheta_{0}<\pi / 2$.

2. Upper and lower bounds for the $\mu_{j}^{m}$ 's. We begin by noting the following inequalities

$$
\frac{1}{3}<\frac{1}{\sin ^{2} \vartheta}-\frac{1}{\vartheta^{2}}<\alpha\left(\vartheta_{0}\right), \quad 0<\vartheta \leq \vartheta_{0} \leq \pi / 2,
$$


where $\alpha(\vartheta)=\sin ^{-2} \vartheta-\vartheta^{2}$ (see [11]). If we multiply (2.1) by $\left(1-4 m^{2}\right) / 4$ with $m \geq 1$, we find

$$
\frac{1-4 m^{2}}{4 \vartheta^{2}}-a_{m}<\frac{1-4 m^{2}}{4 \sin ^{2} \vartheta}<\frac{1-4 m^{2}}{4 \vartheta^{2}}-b_{m}, \quad 0<\vartheta \leq \vartheta_{0}<\pi / 2,
$$

where $a_{m}=\alpha\left(\vartheta_{0}\right)\left(4 m^{2}-1\right) / 4$ and $b_{m}=\left(4 m^{2}-1\right) / 12$.

Next, consider the following differential equations,

$$
\begin{aligned}
& v^{\prime \prime}+\left(\left(\nu+\frac{1}{2}\right)^{2}-a_{m}+\frac{1-4 m^{2}}{4 \vartheta^{2}}\right) v=0, \quad 0<\vartheta<\vartheta_{0}, \\
& u^{\prime \prime}+\left(\left(\nu+\frac{1}{2}\right)^{2}+\frac{1-4 m^{2}}{4 \sin ^{2} \vartheta}\right) u=0, \quad 0<\vartheta<\pi, \\
& V^{\prime \prime}+\left(\left(\nu+\frac{1}{2}\right)^{2}-b_{m}+\frac{1-4 m^{2}}{4 \vartheta^{2}}\right) V=0, \quad 0<\vartheta<\vartheta_{0} .
\end{aligned}
$$

From [10, p. 17], we see that the solutions of $(2.3 \mathrm{a})$ and $(2.3 \mathrm{c})$ are given by

$$
\begin{aligned}
& v=\sqrt{\vartheta} J_{m}\left(\sqrt{\left(\nu+\frac{1}{2}\right)^{2}-a_{m} \vartheta}\right), \\
& V=\sqrt{\vartheta} J_{m}\left(\sqrt{\left(\nu+\frac{1}{2}\right)^{2}-b_{m} \vartheta}\right),
\end{aligned}
$$

respectively, while the solution of $(2.3 \mathrm{~b})$ is easily verified to be $u=\sqrt{\sin \vartheta} P_{\nu}^{m}(\cos \vartheta)$.

In [2], it was shown that $P_{\nu}^{m}(\cos \vartheta)$ has $[\nu-m]$ zeros on $(0, \pi)([x]=n$, when $n \leq x<n+1)$. In the following, we let $\vartheta_{k, \nu}^{m}$ denote the $k$ th zero of $P_{\nu}^{m}(\cos \vartheta)$ on $0<\vartheta<\pi$. In particular, if $\vartheta_{0}=\vartheta_{\nu, k}^{m}$, then $\mu_{k}^{m}\left(\vartheta_{0}\right)=\nu(\nu+1)$. From the Sturm Comparison Theorem [6], it follows that the $k$ th zero of $V$ occurs before the $k$ th zero of $u$, and the $k$ th zero of $u$ occurs before the $k$ th zero of $v$. In particular, we find that

$$
\frac{j_{k}^{m}}{\sqrt{\left(\nu+\frac{1}{2}\right)^{2}-b_{m}}}<\vartheta_{\nu, k}^{m}<\frac{j_{k}^{m}}{\sqrt{\left(\nu+\frac{1}{2}\right)^{2}-a_{m}}},
$$

where $j_{k}^{m}$ is the $k$ th positive zero of the Bessel function $J_{m}(z)$. Arguing in a similar fashion, we find that when $m=0$,

$$
\frac{j_{k}^{0}}{\sqrt{\left(\nu+\frac{1}{2}\right)^{2}-a_{0}}}<\vartheta_{\nu, k}^{0}<\frac{j_{k}^{0}}{\sqrt{\left(\nu+\frac{1}{2}\right)^{2}-b_{0}}} .
$$

We are now ready to establish the main result. 
THEOREM 1. If $\mu_{k}^{m}\left(\vartheta_{0}\right)$ is an eigenvalue of $(*)$ for $0<\vartheta_{0}<\pi / 2$, then

$$
\begin{gathered}
\left(\frac{j_{k}^{m}}{\vartheta_{0}}\right)^{2}+\frac{m^{2}-1}{3}<\mu_{k}^{m}\left(\vartheta_{0}\right)<\left(\frac{j_{k}^{m}}{\vartheta_{0}}\right)^{2}+\frac{4 m^{2}-1}{4}\left(\alpha\left(\vartheta_{0}\right)-\frac{1}{4 m^{2}-1}\right), \\
\left(\frac{j_{k}^{0}}{\vartheta_{0}}\right)^{2}-\frac{1}{4} \alpha\left(\vartheta_{0}\right)<\mu_{k}^{0}\left(\vartheta_{0}\right)<\left(\frac{j_{k}^{0}}{\vartheta_{0}}\right)^{2}-\frac{1}{3},
\end{gathered}
$$

where $\alpha\left(\vartheta_{0}\right)=\sin ^{-2} \vartheta_{0}-\vartheta_{0}^{-2}$.

Proof. The estimates in (2.5a) follow by setting $\nu=\nu_{k}^{m}\left(\vartheta_{0}\right)$ and $\mu_{k}^{m}=\nu_{k}^{m}\left(\nu_{k}^{m}+1\right)$ in (2.4a), squaring the resulting inequalities, replacing $a_{m}$ and $b_{m}$ by their definitions and then simplifying. The estimates in $(2.5 b)$ follow in a similar fashion from $(2.4 b)$.

3. Concluding remarks. The notation in $[4,8]$ is slightly different than that used here $\left(\mu_{1}^{0}=2 \lambda_{1}\right.$ where $\lambda_{1}$ is the first eigenvalue of the Laplacian as defined in [8]; in [4], we also need to set $\bar{u}=\pi-\vartheta_{0}$ and $\left.R=D=1\right)$. Taking the best estimates from [4, 8], we find that $\lambda_{\min }<\mu_{1}^{0}\left(\vartheta_{0}\right)<\lambda_{\max }$ where $\lambda_{\max }=\left(j_{1}^{0} / \vartheta_{0}\right)^{2}$ (see [8]) and $\lambda_{\min }=\left(\pi / \vartheta_{0}\right)^{2}-\frac{1}{2}$ (see [4]). However, the upper bound in (2.5b) is less than $\lambda_{\max }$ by the additive constant $-\frac{1}{3}$. The lower bound in $(2.5 \mathrm{~b})$ is greater than $\lambda_{\text {min }}$, since

$$
\left(\frac{j_{1}^{0}}{\vartheta_{0}}\right)^{2}-\frac{1}{4} \alpha\left(\vartheta_{0}\right) \geq\left(\frac{j_{1}^{0}}{\vartheta_{0}}\right)^{2}-\frac{1}{4} \alpha(\pi / 2)>\left(\frac{\pi}{\vartheta_{0}}\right)^{2}-\frac{1}{2}=\lambda_{\min } .
$$

Moreover, both bounds in (2.5) are asymptotically sharp as $\vartheta_{0} \rightarrow 0$, because (see [11])

$$
\lim _{\vartheta_{0} \rightarrow 0} \vartheta_{0}\left(\nu_{k}^{m}\left(\vartheta_{0}\right)+\frac{1}{2}\right)=j_{k}^{m}
$$

Although Theorem 1 applies to any eigenvalue of $(*)$ when $0<\vartheta_{0}<\pi / 2$, since the lower bound in (2.1) is independent of $\vartheta_{0}$ for $0<\vartheta_{0}<\pi$, it follows that the lower bound for $\mu_{k}^{m}\left(\vartheta_{0}\right)$ with $m \geq 1$ and the upper bound for $\mu_{k}^{0}\left(\vartheta_{0}\right)$ hold for all $0<\vartheta_{0}<\pi$.

\section{REFERENCES}

[1] F. Baginski, Ordering the zeroes of the Legendre functions $P_{\nu}^{m}\left(z_{0}\right)$ when considered as a function of $\nu$, J. Math. Anal. Appl. 147 (1), 296-308 (1990)

[2] F. Baginski, Comparison theorems for the $\nu$-zeroes of Legendre function $P_{\nu}^{\prime \prime}\left(z_{0}\right)$ when $-1<z_{0}<$ 1, Proc. Amer. Math. Soc., to appear

[3] F. Baginski, The buckling of elastic spherical caps, (preprint)

[4] G. Del Grosso, A. Gerardi, and F. Marchetti, A diffiusion model for patch formation on cellular surfaces, Appl. Math. Optim. 7, 125-135 (1981)

[5] E. Hobson. The Theory of Spherical and Ellipsoidal Harmonics. Cambridge University Press. London, 1931

[6] A. Laforgia and M. E. Muldoon. Some consequences of the Sturm comparison theorem. Am. Math. Monthly 93, 89-94 (1986)

[7] F. I. Niordson, Shell Theory. Elsevier Science Publishers B. V.. Amsterdam. 1985

[8] M. Pinsky. The first eigenvalue of a spherical cap. Appl. Math. Optim 7, 137-139 (1981)

[9] J. Smoller, Shock W'aves and Reaction-Diffiusion Equations. Springer-Verlag. New York. 1983 
[10] G. Szegö, Orthogonal Polynomials, American Mathematical Society Colloquium Publications, New York, 1939

[11] G. Szegö, Inequalities for the zeroes of Legendre polynomials and related functions, Trans. Amer. Math. Soc. 39, 1-17 (1936)

[12] H. F. Weinberger, A First Course in Partial Differential Equations with Complex Variables and Transform Methods, Wiley, New York, 1965 